\title{
Hereditary Periodic Fever Syndromes: Autoinflammatory Diseases
}

\begin{abstract}
Key words: Familial Mediterranean fever, hereditory periodic fever syndrome, amyloidosis, pyrin, colchicin
\end{abstract}

\section{Hereditary periodic fever syndromes (HPFs)}

Hereditory periodic fever syndromes (HPFs) consists of four or more genetic diseases characterized by recurrent inflammatory episodes of clinical manifestations, particular, fever and serosal, synovial, and/or cutaneous inflammation. Familial Mediterranean fever (FMF) and the hyper immunoglobulinemia D with periodic fever syndromes (HIDS) are inherited in an autosomal recessive manner and tumor necrosis factor (TNF) receptor-associated periodic syndrome (TRAPS) and Muckle-Wells syndrome (MWS) are dominantly inherited (1). Each disease has distinctive clinical features in the results of recent molecular genetic reports. Since the discovery of the susceptibility gene for FMF in 1997 (2), there has been remarkable progress in the understanding of a category of inflammatory conditions termed HPFs. HPFs have been thought to be extremely rare diseases in Japan. More than 20 years ago, FMF was referred to as "etiocholanone fever" (3). It is likely that Japanese adult patients with HPFs were overlooked. A new pathogenesis of diseases has been elucidated, and a new era of rare and complicated diseases of HPFs has come, and the new developments will bring hope for Japanese patients suffering from HPFs.

\section{Familial Mediterranean fever}

Familial Mediterranean fever (FMF), familial paroxysmal polyserositis is an autosomal recessive inherited disorder, characterized by recurrent episodes of fever, peritonitis, and/or pleuritis. Arthritis, cutaneous lesions, and amyloidosis are seen. FMF occurs predominantly in patients of nonAshkenazi (Sephardic) Jewish, Armenian, and Arabic ancestry. However, patients of Italian, Ashkenazi Jewish, and Anglo-Saxon descent have also been reported. The male : female ratio is 1.5 to $2: 1$. The first episode occurs before the age of 20 years in most patients. The attack lasts 1 to three days and presents as fever with or without abdominal pain, pleuritic chest pain, skin rash, and arthritis (1).

FMF is caused by mutations in $M E F V$, a 10 -exon gene encoded on the short arm of chromosome 16. MEFV codes for a 781-amino acid protein called pyrin, or marenostrin. A total of 28 mutations have been identified, most of them clustered in exon 10. Of the common mutations, M694V is thought be associated with an increased risk of amyloidosis, and E148Q generally has been associated with a mild phenotype (1). The first Japanese case of FMF with $M E F V$ mutation was reported by Shinozaki et al (4) and so far, more than 20 cases have been described. Suzuki et al (5) described a case of FMF with Pyrin E148 mutation.

\section{See also p 765 .}

Pyrin is expressed primarily in neutrophils, eosinophils and cytokine-activated monocytes. Full-length pyrin colocalises with microtubules and the actin cytoskeleton and contains at least four different domains. The PYD, the 160170 aminino acids B30.2 domain (located at the C-terminal segment), and the B-Box and coiled-coil (CC) segments. Most FMF mutations are located in the B30.2 domain but function remains unknown.

The N-terminal pyrin domain (PYD) is homologous to the death domain (DD), death effector domain (DED) and caspase recruitment domain (CARD) subfamilies. It has been found that each domain can interact only with its similar counterpart. A pivotal compound in this system is a protein called ASC (apoptosis-associated speck-like protein with a caspase recruitment domain). This protein has a pyrin domain and a CARD domain. When there is a stimulus for inflammation, ASC interacts with the CARD of procaspase 1 (IL-1 $\beta$ coverting enzyme or ICE) and induces a cascade which leads to the formation of IL- $1 \beta$ and other cytokines that inhibit apoptosis. However, if pyrin interacts with the pyrin domain of ASC, it can no longer interact with the procaspase. Thus, the production of IL- $1 \beta$ is inhibited and normal apoptosis is allowed. Since patients with FMF have the defective gene coding pyrin due to mutation, it would be expected that pyrin interacts poorly with ASC allowing it react with the procaspase, leading to IL- $1 \beta$ production, the inhibition of apoptosis, and enhancement of the inflammatory burst. This is the "apoptosis inhibition" hypothesis (3, 6).

\section{Clinical manifestations of FMF}

The symptoms of FMF often begin between the ages of 5 and 15 , although sometimes onset occurs as late as age 50 . The usual acute episode lasts 24 to 48 hours. The attacks range frequency if from twice weekly to once a year. 1) Fever is a cardinal manifestation. 2) Abdominal pain occurs 
in more than $90 \%$ of patients. The pain usually starts in one quadrant and then spreads to involve the whole abdomen. 3) Pleuritic pain is seen in $25-50 \%$ of patients. 4) Arthritis of large joints. 5) Skin lesion called erysipleloid erythema, a demarcated, raised, painful rash, on the lower leg, ankle or foot (7). Approximately 25 percent of FMF patients in Israel have developed amyloidosis. Death usually results from underlying diseases.

\section{Treatment}

In 1972, Goldfinger (8) showed that the prophylactic use of colchicines dramatically reduces the number of attacks. The administration of colchicine regularly is at the optimum doseage of 1.5-2.0 mg/day. The drug can also induce the regression of amyloid deposits to a certain extent. NSAIDs are preferable for the attack period.

Other candidates are thalidomide and anti-TNF agents. Prevention of cold-associated acute inflammation in familial cold autoinflammatory syndrome by interleukin-1 receptor antagonist has been reported (9).

Shigeto KoBAYASHI, MD, PhD Division of Rheumatology, Department of Internal Medicine, Juntendo Koshigaya Hospital, 560 Fukuroyama, Koshigaya, Saitama 279-0032

\section{References}

1) Kastner DL. Periodic syndrome. Muscloskeletal signs and symptoms in: Primer on the Rheumatic Diseases. Klippel JH, Stone JH, Weyand CM, Eds ed 12, Arthritis Foundation, Georgea, 2001: 194-201.

2) The International FMF Consortium. Ancient missense mutations in a new member of the RoRet gene family are likely to cause familial Mediterranean fever. Cell 90: 797-807, 1997.

3) Tunca M, Ben-Chetrit E. Familial Mediterranean fever in 2003. Pathogenesis and management. Clin Exp Rheumatol 21 suppl 30: s49s52, 2003

4) Shinozaki K, Agematsu K, Yasui K, et al. Familial Mediterranean fever in 2 Japanese families J Rheumatol 29: 1324-1325, 2002.

5) Suzuki T, Nakamura A, Yazaki M, Ikeda SI. A Japanese case of familial Mediterranean fever with homozygosity for the Pyrin E148Q mutation. Intern Med 44: 765-766, 2005.

6) McDermott MF. A common pathway in periodic fever syndromes. Trends Immunol 25: 457-460, 2004.

7) Pras M, Kastner DL. Familial Mediterranean fever. in: Rheumatology. Klippel JH, Dieppe PA Eds. 2nd Ed. 5.23.1-23.4, Mosby, London, 1998.

8) Goldfinger SE. Colchicine therapy for familiar Mediterrane fever. N Engl J Med 291: 934-937, 1974

9) Hoffman HM, Rosengren S, Boyle D, et al. Prevention of coldassociated acute inflammation in familial cold autoinflammatory syndrome by interleukin-1 receptor antagonist. Lancet 364: 1779-1785, 2004. 